\title{
PROGRESS TOWARD USING MRI AND A HEART MODEL TO ESTIMATE PATIENT-SPECIFIC INDICES OF CARDIAC FUNCTION
}

\author{
M. Sermesant ${ }^{1}$, P. Moireau ${ }^{2}$, R. Andriantsimiavona ${ }^{1}$, J. Sainte-Marie ${ }^{2}$, \\ O. Camara ${ }^{1}$, R. Cimrman ${ }^{3}$, D.L.G. Hill ${ }^{1}$, D. Chapelle ${ }^{2}$ and R. Razavi ${ }^{1}$
}

\begin{abstract}
In this article, we present a framework to estimate cardiac function parameters like local myocardium contractility using clinical MRI, a heart model and data assimilation. First, we build a generic anatomical model of the ventricles including muscle fibre orientations and anatomical subdivisions. Then, this model is deformed to fit a segmented MRI, using an affine registration method and a local deformable biomechanical model. An electromechanical model of the heart can be simulated on this mesh. Data assimilation makes it possible to estimate local contractility from given displacements. Presented results on simulated data and adjustment to clinical data are very promising. Current work on model calibration and estimation of patient parameters open up possibilities for clinical application of this framework.
\end{abstract}

Résumé. Dans cet article, nous présentons un cadre de travail pour estimer certains paramètres de la fonction cardiaque (comme la contractilité locale) en utilisant l'IRM, un modèle du myocarde et l'assimilation de données. Tout d'abord, nous détaillons la construction d'un modèle générique du myocarde incluant l'orientation des fibres musculaires et les différents segments anatomiques. Ce modèle peut être déformé pour s'ajuster aux images cliniques. Un modèle électromécanique du myocarde est ensuite présenté et simulé. Enfin, l'assimilation de données permet d'estimer la contractilité locale à partir de données telles que les déplacements. Les résultats sur l'ajustement du modèle à la géométrie du patient et l'assimilation sur des données simulées sont très encourageants. Les travaux en cours sur la calibration du modèle et l'estimation de paramètres du patient ouvrent de nouvelles possibilités pour l'application de ce cadre de travail dans un environnement clinique.

\section{INTRODUCTION}

The integration of knowledge from biology, physics and computer science makes it possible to combine in vivo observations, in vitro experiments and in silico simulations. From these points of view, knowledge of the heart function has greatly improved at the nanoscopic, microscopic and mesoscopic scales [10,12].

Due to the limitations of medical imaging, modelling capabilities and computational power, the validation of heart models with human in vivo data and furthermore their use in clinical applications are very challenging. We present in this paper a framework aiming at overcoming these difficulties by directly combining modelling of the heart, cardiac function estimation and parameter adjustment. The detailed application is the estimation

\footnotetext{
1 Cardiac MR Research Group, King's College London, 5th Floor Thomas Guy House, Guy's Hospital, London, UK.

2 MACS project, INRIA Rocquencourt, France.

3 New Technologies Research Centre, Západočeská univerzita v Plzni, Univerzitní 22, 30614 Plzeň, Czech Republic.

Corresponding author: derek.hill@ieee.org
}

(C) EDP Sciences, SMAI 2005 
of local contractility in the myocardium from displacements measured in medical images. Physiologically, myocardium contractility represents the ability of the heart muscle to force blood through its chambers. Estimating myocardium local contractility is of high clinical impact: it could make it possible to achieve an early diagnosis of some cardio-myopathies, like ischemic areas, compared to global indices. Moreover, it provides an excellent tool for evaluating the efficiency of local therapies like stem cells, or plan revascularisation interventions, to target viable myocardium.

The five components presented in this paper are: medical imaging techniques to observe the heart in vivo, construction of a generic anatomical model, equations used to simulate the cardiac electromechanical behaviour, adjustment of a generic heart model to patient anatomy, and data assimilation method to estimate local contractility. We emphasise in each of these sections the advances made and the difficulties encountered.

\section{Modalities of Observation}

Magnetic Resonance Imaging (MRI) is a successful and promising modality but it remains difficult to use, and is seldom used outside major research centres. A particular challenge in Cardiac MRI is that the heart is a moving organ, in a moving environment (breathing). This restricts the resolution that can be obtained, and often leads to inconsistent data, making subsequent analysis challenging.

\subsection{Anatomical Imaging}

Black-Blood Imaging is characterised by the suppression of the signal from flowing blood. This gives a good visualisation of the myocardium, which is of great interest for our modelling purpose. Unfortunately, due to the pre-pulse and the inversion time, black-blood imaging is essentially a single slice sequence for each breath-hold. However, new methods are emerging to allow multi-slice imaging.

Bright-Blood Imaging at the contrary generates high signal intensity for blood and can be used for dynamic (cine) images of a small number of slices in each breath-hold. Therefore, bright-blood acquisitions allow both morphological and functional assessment. A major drawback however is that delineating the epicardium remains difficult due to the poor contrast between the heart and the lungs. 4D images with bright blood are becoming available to provide approximately isotropic resolution. These sequences usually have inferior temporal resolution and contrast to $2 \mathrm{D}$ dynamic sequences.

\subsection{Functional Imaging}

Global Cardiac Function Analysis. The quantification of ventricular volumes, myocardial mass and ejection fraction using MRI are both accurate and reproducible in the hand of experienced users. However, the time required for acquisition and analysis of MR images hamper the MR exams to enter clinical routine. With lengthy exam, the constraint of multiple breath holding adds to the patient discomfort.

Fractional k-space filling methods or view-sharing strategies enable the imaging time to be considerably reduced without substantial loss of image quality and resolution. However, these techniques are mainly used for $2 \mathrm{D}$ imaging. The major issue with multiple $2 \mathrm{D}$ imaging is that the coverage of the ventricles requires multiple breath-holds. Inconsistencies in the different breath-hold positions can lead to errors in image interpretation

3D multiphase single breath-hold imaging methods appear to be a promising alternative for functional imaging. Nevertheless, compromises had to be made in terms of image quality, spatial and temporal resolutions.

Regional Cardiac Function Analysis. Ejection Fraction is a global parameter that assesses the status of the cardiac function with great efficiency. However, it is not specific enough for myocardial efficiency and contractility. The study of wall deformations provides more insights on the mechanical contraction of the heart. Tagging is a well-known method to track local deformations of a "printed" grid as it follows the heart contracting. It enables parameters such as twist, strain and strain rate to be derived. The extraction of motion is based on models or registration techniques [3]. Although accurate, it is time consuming and suffer from the low spatial resolution of tags. More recently, the development of harmonic phase (HARP) and displacement 
encoding stimulated echo (DENSE) methods makes it possible to quantify the displacement of each moving pixel inside the myocardium. These techniques though are currently limited to 2D displacements.

The direct relationship between myocardial motion and contractility is difficult to estimate directly from the images. A model-based approach could thus help to extract this hidden information.

\section{Generic Anatomical Heart Model}

The aim of this work is to provide a method for model-based analysis of the previously described medical images. The idea is to adjust a biomechanical model of the myocardium using these images in order to extract hidden parameters useful for diagnosis, like local contractility. To achieve the simulation of cardiac electromechanical activity, we need the myocardium geometry and the muscle fibre orientations as anatomical inputs. The geometry gives the domain on which to carry out computations. Fibre orientations are important for both the active and passive behaviour of the myocardium.

The difficulty for this step is to obtain both types of information for a particular myocardium. On the one hand, geometry can be extracted from anatomical medical images. But fibre orientations cannot be measured in vivo and current diffusion tensor images of fixed hearts are still noisy compared to the smoothness required by the electromechanical computations.

On the other hand, when fibre orientations are measured from dissection, the geometry is often not available, or in so deformed shape that adjustment of the model to the in vivo images becomes very challenging.

Due to these problems, our approach is to synthesise a generic anatomical model of the myocardium, composed of a simple geometry, close enough to in vivo observations, and of synthetic fibre orientations, generated according to the measurements available in the literature.

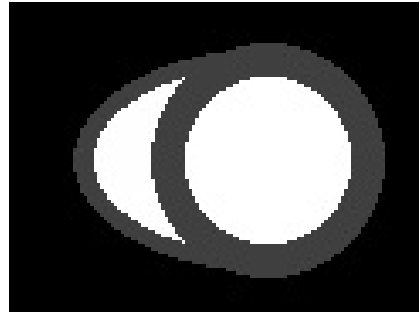

(a)

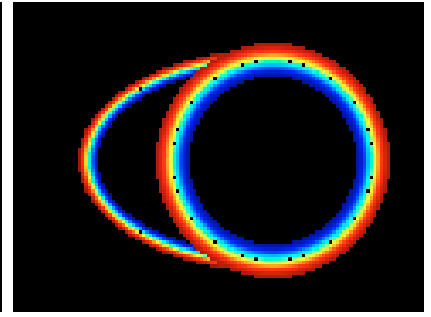

(b)

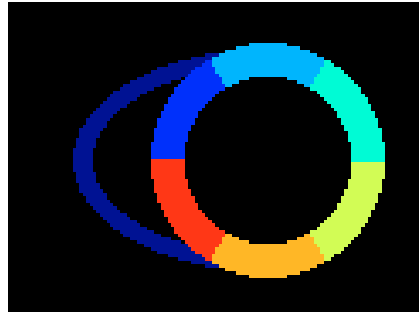

(c)

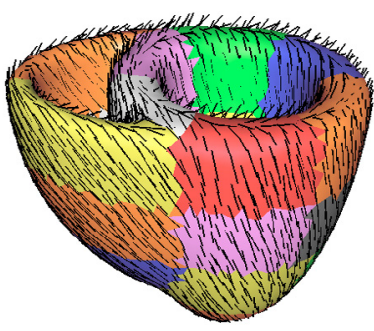

(d)

FIGURE 1. Generic anatomical heart model: equatorial short axis slice of (a) geometry, (b) fibres orientation (elevation angle), (c) AHA division. (d) Resulting mesh with fibre orientations (black segments) and AHA divisions (colours).

Heart Geometry. Left ventricle shape is close to a truncated ellipsoid, this approximation is the one generally used for left ventricle estimation from $2 \mathrm{D}$ images. The right ventricle can also be approximated by a truncated ellipsoid. The generic heart model geometry is defined using different parameters for the radii of the left and right ventricles ellipsoids, their thickness and the height of the truncating basal plane (see Fig. 1).

Heart Fibres Orientations. It is well known that muscle fibre orientations vary across the myocardial wall. In our heart model, the elevation angle between the fibre and the short axis plane varies between +90 and -90 degrees from the endocardium to the epicardium. It is in good agreement with data available in the literature from dissection or diffusion tensor MR.

Cardiac Anatomical Divisions. Accurate calibration, estimation and analysis of the model is made easier by subdividing it into different anatomical regions. Generating the model makes it possible to analytically divide it into the 17 regions of interest proposed by the American Heart Association (AHA). 
Myocardium Mesh. From the anatomical image, a triangulated surface is extracted using the marching cubes algorithm. This surface is used to create a tetrahedral mesh with the INRIA GHS3D software ${ }^{1}$. Finally, fibre orientations and subdivisions are assigned to the mesh using rasterization, see details in [16]. The resulting mesh is presented Fig. 1d.

\section{Cardiac Muscle Biomechanics}

Modelling the myocardium behaviour is difficult because of its active, non-linear, anisotropic nature. Several constitutive laws were proposed for the active and passive properties of the myocardium [10, 12].

\subsection{Myofibre Active Constitutive Law}

A constitutive law of the electrically-activated myofibres was proposed by Bestel-Clément-Sorine [2]. Whereas most modelling endeavours rely on heuristic considerations, this law is based on a multi-scale approach taking into account the behaviour of myosin molecular motors, and the resulting sarcomere dynamics is in agreement with the sliding filament hypothesis introduced in [8]. Denoting by $\sigma_{c}$ the active stress and by $\varepsilon_{c}$ the strain along the sarcomere, this law relates $\sigma_{c}$ and $\varepsilon_{c}$ as follows:

$$
\begin{cases}\dot{\tau}_{c}=k_{c} \dot{\varepsilon}_{c}-\left(\alpha\left|\dot{\varepsilon}_{c}\right|+|u|\right) \tau_{c}+\sigma_{0}|u|_{+} & \tau_{c}(0)=0 \\ \dot{k}_{c}=-\left(\alpha\left|\dot{\varepsilon}_{c}\right|+|u|\right) k_{c}+k_{0}|u|_{+} & k_{c}(0)=0 \\ \sigma_{c}=\tau_{c}+\mu \dot{\varepsilon}_{c}+k_{c} \xi_{0} & \end{cases}
$$

where $u$ represents the electrical input $\left(u>0\right.$ : contraction, $u \leq 0$ : relaxation). Parameters $k_{0}$ and $\sigma_{0}$ characterise muscular contractility and respectively correspond to the maximum value for the active stiffness $k_{c}$ and for the stress $\tau_{c}$ in the sarcomere, while $\mu$ is a viscosity parameter.

For computational reasons, we use simplified activation patterns such as uniform activation (in space) or a planar wave travelling from apex to base to represent the propagation of the action potential.

\subsection{D Model of the Myocardium}

The above active constitutive law was used within a rheological model of Hill-Maxwell type [4], as depicted in Fig. 2a. The element $E_{c}$ accounts for the contractile electrically-activated behaviour governed by (1). In addition, an elastic material law is considered for the series element $E_{s}$, while $E_{p}$ is taken viscoelastic. Based on experimental results, the corresponding stress-strain laws are assumed to be of exponential type for $E_{p}$ [18], and linear for $E_{s}[13]$.

This rheological model is compatible with large displacements and strains and led to a continuum mechanics description of the cardiac tissue [5]. A study and simulations of a simplified 1D model derived from this continuum mechanics model are detailed in [4].

\subsection{Modelling the Blood}

The blood inside each ventricle is modelled as a pressure / volume system. The phases of the cardiac cycle (isovolumetric contraction, ejection, isovolumetric relaxation, filling) are distinguished through coupling conditions between the internal fluid and other parts of the cardiovascular system, namely the atrial cavities and the external circulation. With $P_{\mathrm{v}}, P_{\text {ar }}$ and $P_{\text {at }}$ denoting the blood pressures in the ventricle, the artery, and the atrium, respectively, the ejection occurs when $P_{\mathrm{v}} \geq P_{\mathrm{ar}}$ whereas the mitral valve opens when $P_{\mathrm{v}} \leq P_{\mathrm{at}}$, see Fig. 2b. Denoting by $Q$ the outgoing flow, the coupling conditions can be formulated as a (double) contact problem:

$$
\left\{\begin{array}{lll}
Q \geq 0 & \text { when } P_{\mathrm{v}}=P_{\mathrm{ar}} & \text { ejection } \\
Q=0 & \text { when } P_{\mathrm{at}}<P_{\mathrm{v}}<P_{\mathrm{ar}} & \text { isovolumetric phases } \\
Q \leq 0 & \text { when } P_{\mathrm{v}}=P_{\mathrm{at}} & \text { filling }
\end{array}\right.
$$

\footnotetext{
$1_{\text {http://www-rocq.inria.fr/gamma/ghs3d/ghs.html }}$
} 
To avoid numerical difficulties, we used a regularised form of this function as depicted by the solid line in Fig. 2c. External circulation is modelled by a Windkessel model [17], and blood flow coming from the atria by a pressure $P_{\text {at. }}$.

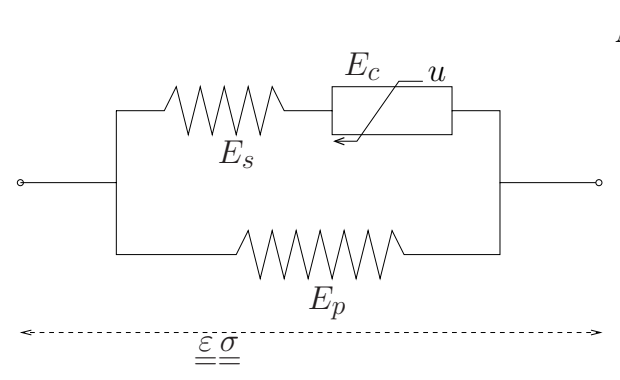

(a)

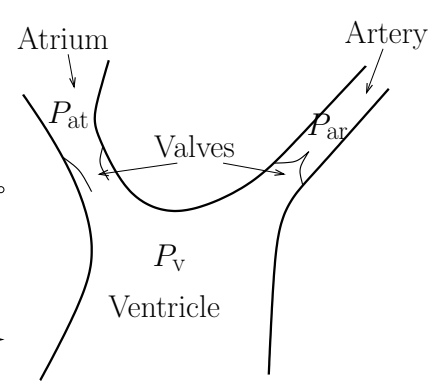

(b)

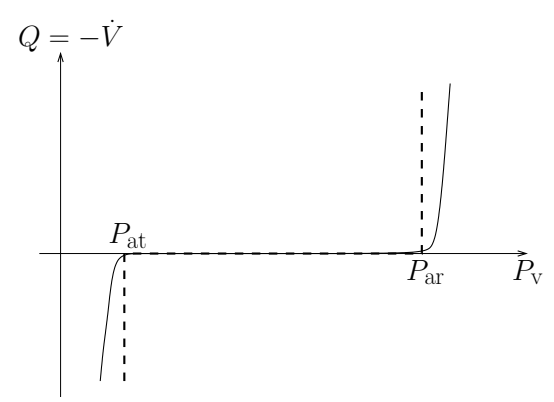

(c)

Figure 2. (a) Hill-Maxwell rheological model. (b) Aortic valve model mechanism. (c) Formulation as a double contact problem, dashed: relation (2), solid: regularised.

\subsection{Numerical Simulations}

Combining all the above modelling components with adequate numerical techniques we obtained numerical simulations of complete heart beats. Some corresponding numerical results concerning commonly-used medical indicators of the heart function are displayed in Figure 3 and they are in good agreement with physiological values. These numerical simulations are then used in order to confront the modelling and the data, namely within a data assimilation procedure.

\section{Data Assimilation}

The aim of data assimilation is to incorporate measurements into a dynamic system model in order to produce accurate estimates of the current (and possibly future) state variables, parameters, initial conditions and input of the model.

The symbol $H$ denoting the observation operator, $Y(t)$ the available measurements and $X(t)$ the model response, the general objective of data assimilation is the minimisation of a cost function $J$ performed over the set of parameters to be estimated

$$
J=\int_{I}\|Y(t)-H X(t)\|_{\Omega}^{2} d t+\text { penalty }
$$

$\|.\|_{\Omega}$ being a suitable norm associated with the problem formulation.

If $I$ denotes the complete simulation time interval $\left[t_{0}, T\right]$, the assimilation technique is said to be variational and corresponds to an optimal control problem $[6,11]$. If at each time step $t_{k}, I=\left[t_{0}, t_{k}\right]$, then the filtering technique is said to be sequential [9].

Due to the complexity of the model and to observability considerations, estimating all the quantities appearing in the complete electromechanical problem is out of reach. Hence we focus on parameters that are crucial for medical purposes in order to detect contraction troubles, in particular the parameters $\sigma_{0}$ and $k_{0}$.

Preliminary results in data assimilation have been presented in [15]. Results presented here have been obtained using numerically simulated observations assimilated with the complete 3D problem on a left ventricle model (Fig. 4).

Both sequential and variational techniques have been tested. Without considering the computational costs, the results given by the two methods are very similar. The current variational method implementation does not 

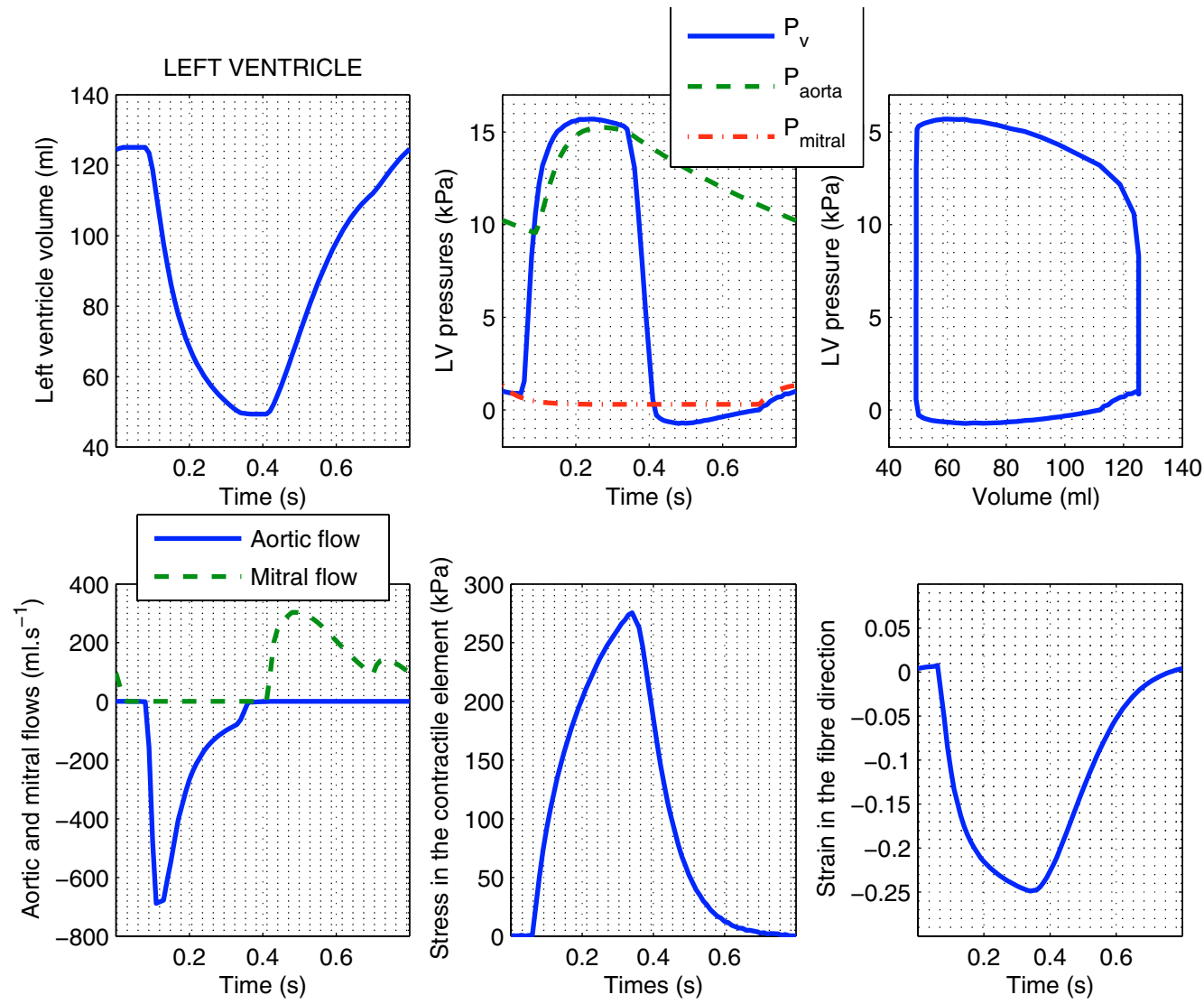

Figure 3. Computed medical indicators (from left to right and top to bottom): left ventricle volume; blood pressures; pressure-volume diagram; aortic and mitral flows; contractile stress and strain along the fibre.

make it possible to estimate time dependent parameters, but this in not our goal in the presented work. The data assimilation process validation is the following:

- The direct 3D problem is simulated with a given parameter $\sigma_{0}$.

- Observations $\left\{Y\left(M_{k}, t_{p}\right)\right\}_{k, p}$ are obtained using $Y\left(M_{k}, t_{p}\right)=H X\left(M_{k}, t_{p}\right), H$ being the chosen observation operator. Here we consider only 30 displacements for random points of the mesh and we add gaussian noise (5\%).

- Starting from a given parameter $\hat{\sigma}_{0}$ different from the one used for the direct simulation, the data assimilation is carried out.

For this simulation we chose $\sigma_{0}(M)$ constant in each of the three regions visible on Fig. 4 a. The result of the estimation of $\sigma_{0}$ is shown in Fig. 4b. The data assimilation process, initialised with a homogeneous distribution, recovered the spatial variations of $\sigma_{0}$ rather accurately. We used the variational technique (four iterations), briefly described in Appendix. The convergence of the parameters estimation for the three regions is presented in Fig. 5

We also compared the performances of variational and sequential procedures (see e.g. [7] for sequential data assimilation techniques) using a simplified heart model. Our preliminary results indicate that sequential algorithms are more effective and robust when estimating several unknown parameters. We show, for example, the convergence in the sequential estimation of two mechanical parameters (namely, the stiffness and viscosity 


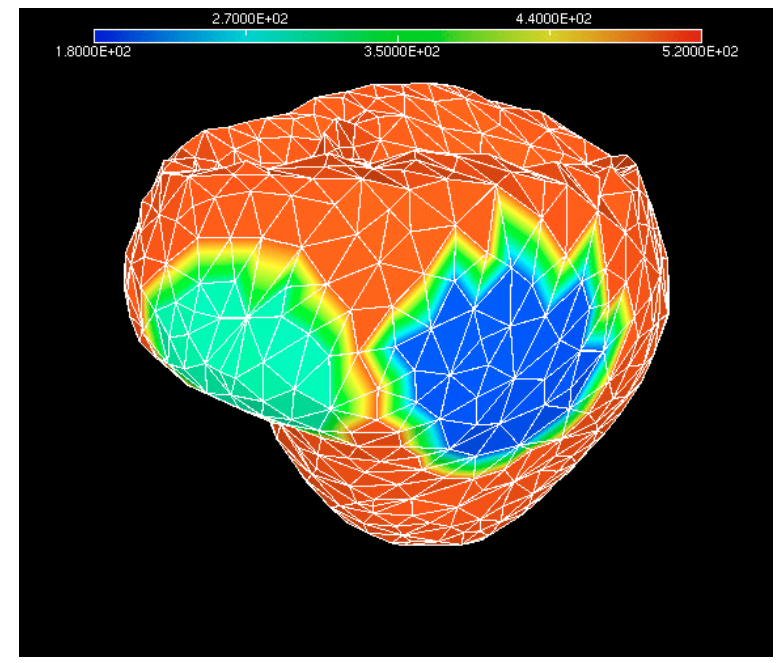

(a) Reference $\sigma_{0}$

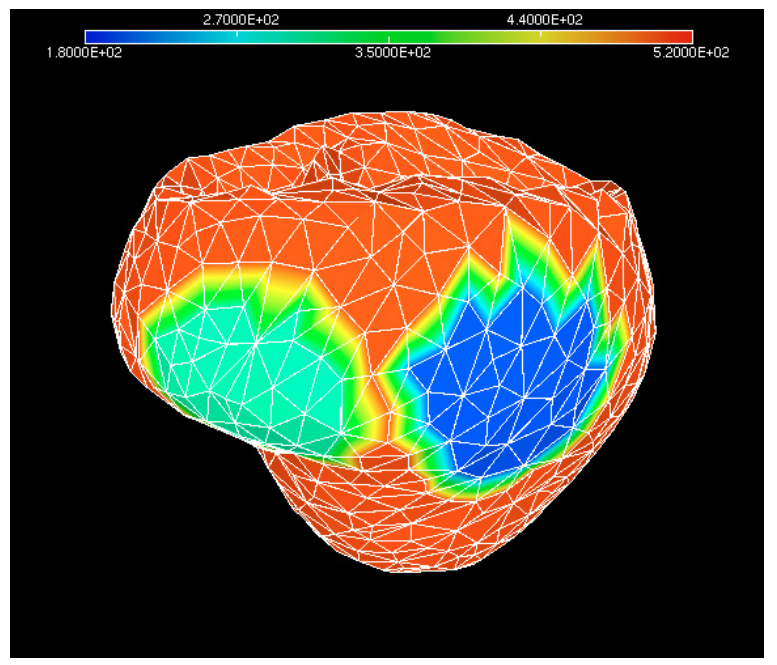

(b) Estimated $\sigma_{0}$

Figure 4. Contractility $\left(\sigma_{0}\right)$ estimation from simulated data. The observations are the displacements on the epicardial and endocardial surfaces and the variational technique is used. The lower contractility region is well recovered by the data assimilation.

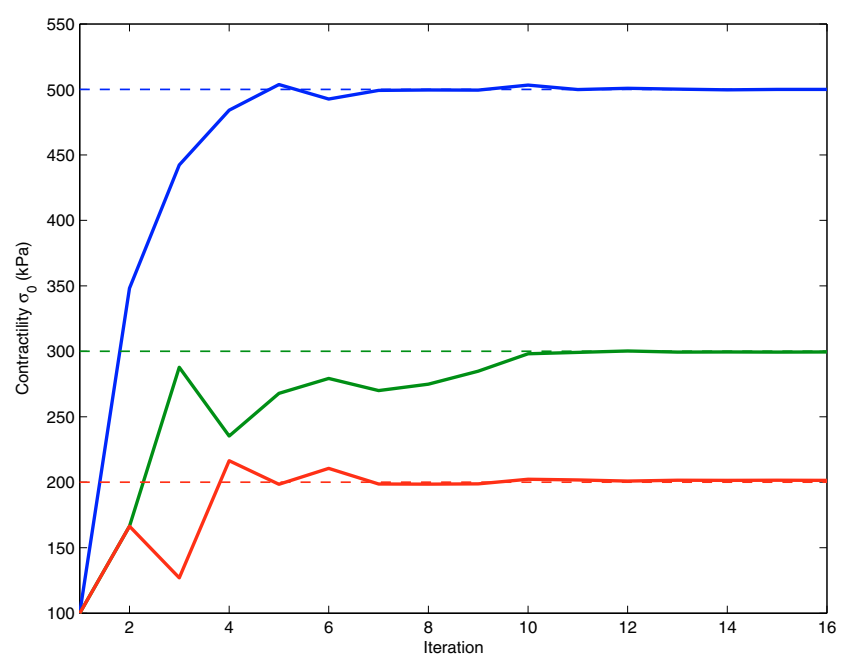

Figure 5. Convergence of contractility parameter $\sigma_{0}$ in the three different regions, using variational assimilation on heart model.

coefficients in the passive branch) in Fig. 6, where we see that the combined estimation converges with excellent accuracy within a fraction of heart beat. When using a variational procedure, similar accuracy can be obtained, albeit only after thirty gradient iterations (over a complete heart cycle for each of them). However, for the complete model, standard extended Kalman filtering is not tractable because of the size of the covariance matrix (namely, the combined size of the state and parameter vectors). We thus currently pursue the development of improved - in terms of effectiveness, robustness and cost - sequential algorithms adapted to the specificities of the heart system. 

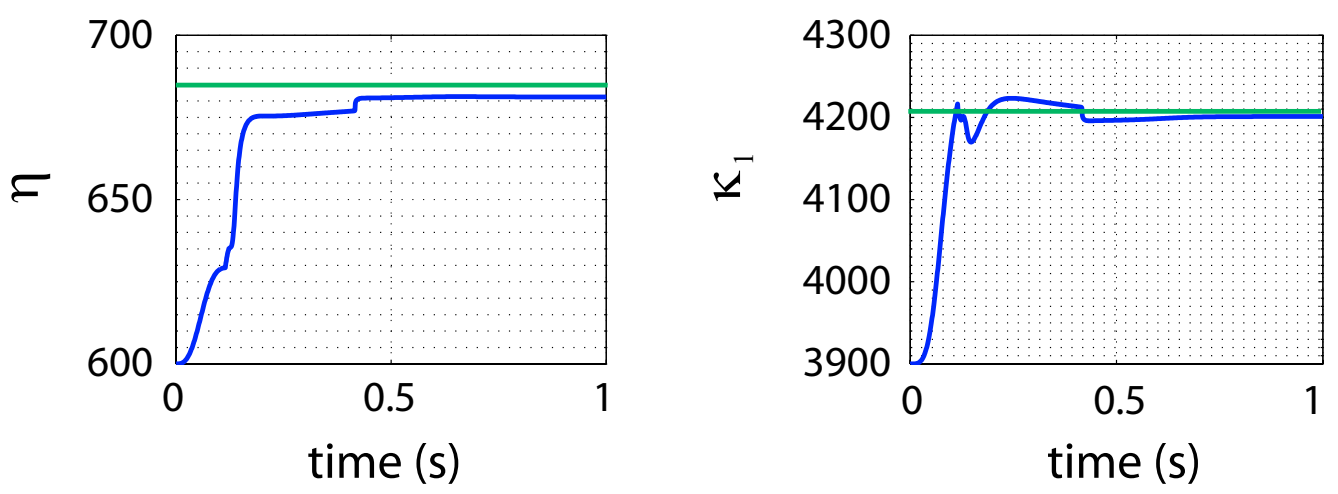

Figure 6 . Convergence of viscosity $(\eta)$ and stiffness $\left(\kappa_{1}\right)$ parameters of the viscoelastic passive branch $E_{p}$ in sequential assimilation on simplified heart model.

\section{Toward Patient-Specific Cardiac Function Estimation}

We demonstrated in the previous section that we could estimate local contractility in the myocardium using sparse known displacements and a biomechanical model, through data assimilation. Before being able to run this method on clinical data, we first have to adjust the model anatomy to the patient anatomy.

\subsection{Patient-specific Heart Anatomy}

Automatic segmentation of the myocardium from MRI is still very difficult, as the epicardium is not easily distinguished and the right ventricle is quite thin. Our approach is to deform the generic anatomical model designed in Section 3 into the clinical image. This is done in three steps: segmentation of the clinical image blood pools, intensity-based registration for the affine adjustment and deformable model-based segmentation for local deformations.

As the clinically used MR sequences produce relatively homogeneous blood pool intensity, one can semiautomatically segment the ventricular blood pools using a combined boundary-based and regional-based fuzzy classification method [1], in order to ease the registration step.

Then, an automatic affine (15 parameters) registration algorithm is applied from the segmented image to the blood pools from the model geometry, using the cross correlation as similarity measure.

Finally, local adjustment is done with a deformable biomechanical model [16], using the affine transformation computed previously for initialisation. In the deformable model framework, a model evolves under the influence of an internal energy for regularisation, and an external energy to fit it to the image boundaries. We use a volumetric model with visco-elastic properties for the internal energy. Compared to classical surface deformable model, with only geometric regularisation, this biomechanical control preserves surface smoothness and element numerical quality. The aspect ratio mean (resp. standard deviation) are for the original mesh: 0.674 (0.109), after affine transformation: 0.650 (0.113), and after local deformation: $0.652(0.114)$ ). This hierarchical procedure allows precise adjustment of the generic anatomical model to the patient image (Fig. 7)

The deformed geometry integrates the synthetic fibres generated. There is currently no method to measure fibre orientations in vivo. On-going studies on excised hearts about the statistics of the fibre orientations will help quantifying how much these orientations vary from one heart to another. This will make it possible to estimate the error introduced by using generic orientations.

This patient-specific model can then be used for the data assimilation procedure, but there are requirements on the observations used. 


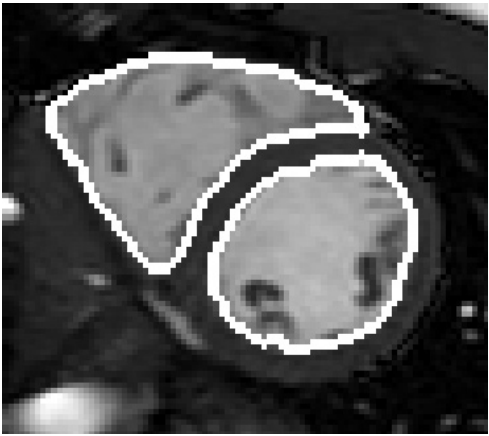

(a)

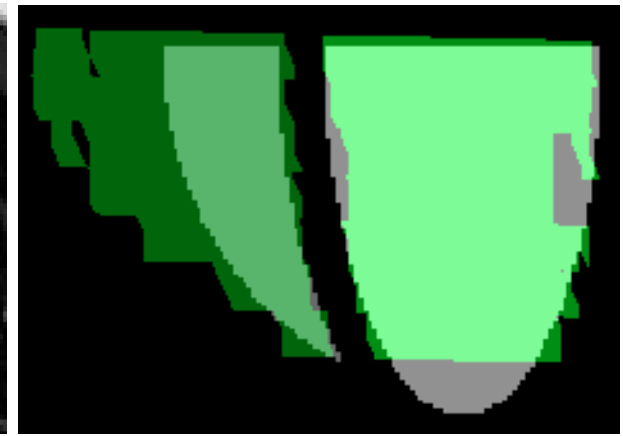

(b)

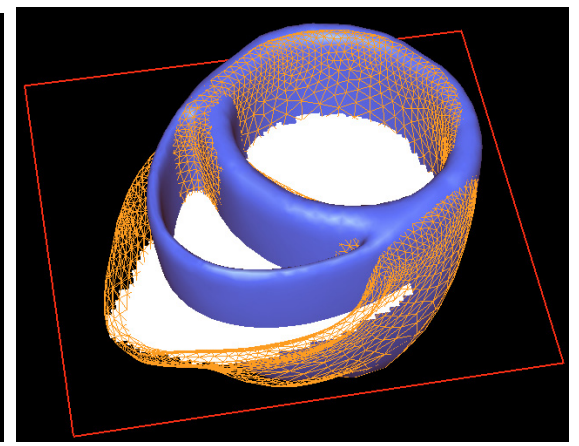

(c)

Figure 7. Patient-specific model three steps: (a) semi-automatic segmentation of the blood pools (white contours), (b) affine registration between the model blood pools (grey) and the segmentation (green), (c) local adjustment (orange wire-frame)from the affine transform of the model (blue surface) using a deformable biomechanical model.

\subsection{Data Assimilation Difficulties}

Measurements used to apply the data assimilation in section 5 are displacements in some points of the myocardium. The idea is to use the same data assimilation procedure, with displacements from tagged MRI.

In data assimilation, difficulties arise from various areas, from the available measurements to the complexity of the operator (type of variables, dimension, rank) and the natures of the spaces and norms used. Current work on these difficulties should help design the best possible operators to achieve this parameter estimation.

A difficulty is the invertibility of this observation operator because we want to obtain the state $X$ and the parameters from the observations using a generalised inverse of $H$. The analysis of this invertibility property (observability) is very difficult in general both as regards surjectivity (whether there exists a set of parameters and variables which leads to the given observation) and injectivity (whether this set is unique).

Another difficulty in the choice of the observation operator lies in the fact that the efficiency of the filtering technique is highly dependent on the noise. To avoid adding noise in the different image processing steps, an idea could be to formulate an operator as close to the measurements as possible. We could consider the tagged images as the observation by simulating tagged images from the model displacements. Recent full 3D tagged MRI could allow direct 3D comparison [14].

\section{Conclusion}

We presented in this article a framework and first results toward automatic estimation of local contractility from MRI and a model of the myocardium. We detailed the medical images used and the information we can extract from it, the construction of a generic anatomical model of the ventricular myocardium integrating muscle fibre orientations and its subdivision into segments, the biomechanical modelling of the myocardium, and a data assimilation method to automatically adjust the parameters of the model from known displacements.

We demonstrated the capability of such a framework, with also pointing out the different difficulties at the theoretical and practical levels. The results so far obtained by confronting modelling and clinical data are very promising. Precise calibration of the model before data assimilation is difficult and additional measurements can help for this task. Progresses in MRI, especially in flow measurements, can help in obtaining patient-specific boundary conditions.

Current work on observation operators should help in its choice. For instance, whether the observation operator can be written in a Lagrangian framework, as with tagged MRI. Lagrangian approach is more easily dealt with, but it is not suitable for all types of measurements, in particular for those directly related to the 
deformed geometry. Many clinical observations (e.g. cine MRI and ultrasound) are indeed more Eulerian in essence.

Future developments are planned to integrate different modalities. For instance, with patients undergoing electrophysiology studies, electrophysiology clinical data can be acquired, using XMR interventional imaging for instance. Such datasets open up possibilities to also adjust electrophysiology models. We point out that an interesting open problem concerns whether or not the electrical activity may also be estimated from displacements measurements of the myocardium. The proposed framework could give insights on this problem. Finally, coupling models and parameter estimation is valuable for intervention planning and therapy testing, owing to the predictive capacity of modelling.

\section{ACKNowledgements}

The authors would like to thank for their collaboration the Cardiac MR Research Group in Guy's Hospital, London and the co-workers of the ICEMA collaborative research actions ${ }^{2,3}$ funded by INRIA. Part of this work was done during the Summer Mathematical Research Centre on Scientific Computing and its Applications (CEMRACS $)^{4}$. The authors acknowledge grant support from EPSRC (M.S., O.C. and R.A.) and the use of software developed by the Epidaure project ${ }^{5}$, INRIA.

\section{APPENDIX}

Variational data assimilation techniques are based on an iterative approximation of the optimality condition $\nabla_{S} J\left(S^{*}\right)=0$, where $S$ denotes the parameter set to estimate, leading to an adjoint problem. If the problem to solve is (A), in the absence of a penalty term in $J$, the adjoint state $P$ is governed by (B):

$$
\text { (A) }\left\{\begin{array} { l } 
{ \dot { X } = F ( X , S , t ) } \\
{ X ( t _ { 0 } ) = X _ { 0 } } \\
{ S \text { unknown parameters } }
\end{array} \quad \text { (B) } \left\{\begin{array}{l}
\dot{P}+\left[\frac{\partial F}{\partial X}\right]^{t} P=H^{t}(H X-Y) \\
P(T)=0
\end{array}\right.\right.
$$

The numerical algorithm used is the following:

- Start from a first guess $S_{0}$ of the parameter set

- Start iteration $n$

- Integrate the direct model from 0 to $T$

- Integrate the adjoint model from $T$ to 0

- Calculate the gradient $\nabla J\left(S_{n}\right)=\int_{0}^{T}\left[\frac{\partial F}{\partial S_{n}}\right] P d t$

- Compute $S_{n+1}=S_{n}+\rho_{n} \nabla J\left(S_{n}\right)$

- $n \rightarrow n+1$ until a stopping criterion on $J$ is reached

This algorithm was used to make the local contractility evolve (with $S=\sigma_{0}(M)$ ) in the data assimilation results presented in section 5 .

\section{REFERENCES}

[1] R. Andriantsimiavona, L. Griffin, D. Hill, and R. Razavi. Simple cardiac MRI segmentation. In International Society for Magnetic Resonance in Medicine Scientific Meeting, volume 6, page 951, 2003.

[2] J. Bestel, F. Clément, and M. Sorine. A biomechanical model of muscle contraction. In Medical Image Computing and Computer-Assisted intervention (MICCAI'01), volume 2208 of Lecture Notes in Computer Science (LNCS), pages 1159-1161. Springer, 2001.

\footnotetext{
${ }^{2}$ http://www-rocq.inria.fr/who/Frederique.Clement/icema.html

3 http://www-rocq.inria.fr/sosso/icema2/icema2.html

${ }^{4}$ http://smai.emath.fr/cemracs/cemracs04/index.php

${ }^{5}$ http://www-sop.inria.fr/epidaure/index.php
} 
[3] R. Chandrashekara, R. Mohiaddin, and D. Rueckert. Analysis of 3-D myocardial motion in tagged MR images using nonrigid image registration. IEEE Transactions on Medical Imaging, 23(10):1245-1250, 2004.

[4] D. Chapelle, F. Clément, F. Génot, P. Le Tallec, M. Sorine, and J. Urquiza. A physiologically-based model for the active cardiac muscle contraction. In Functional Imaging and Modeling of the Heart (FIMH'01), number 2230 in Lecture Notes in Computer Science (LNCS), pages 128-133. Springer, 2001.

[5] D. Chapelle, J. Sainte-Marie, and R. Cimrman. Modeling and estimation of the cardiac electromechanical activity. In Proceedings of the ECCOMAS 2004 Conference, 2004.

[6] P. Courtier and O. Talagrand. Variational assimilation of meteorological observations with the adjoint vorticity equation. Quart. J. Roy. Meteorol. Soc., 113:1311-1347, 1987.

[7] G. Evensen, D. Dee, and J. Schröter. Parameter estimation in dynamical models. In E. Chassignet and J. Verron, editors, Ocean Modeling and Parameterizations, NATO ASI. Kluwer Academic, 1998.

[8] A.F. Huxley. Muscle structure and theories of contraction. Progress in Biophysics E Biological Chemistry, 7:255-318, 1957.

[9] R.E. Kalman. A new approach to linear filtering and prediction problems. ASME Trans.-Journal of Basic Engineering, 82(Series D):35-45, 1960.

[10] T. Katila, I. Magnin, P. Clarysse, J. Montagnat, and J. Nenonen, editors. Functional Imaging and Modeling of the Heart (FIMH'01), number 2230 in Lecture Notes in Computer Science (LNCS). Springer, 2001.

[11] J.L. Lions. Contrôle optimal des systèmes gouvernés par des équations aux dérivées partielles. Dunod, 1968.

[12] I. Magnin, J. Montagnat, P. Clarysse, J. Nenonen, and T. Katila, editors. Functional Imaging and Modeling of the Heart (FIMH'03), number 2674 in Lecture Notes in Computer Science (LNCS). Springer, 2003.

[13] I. Mirsky and W.W. Parmley. Assessment of passive elastic stiffness for isolated heart muscle and the intact heart. Circul. Research, 33:233-243, 1973.

[14] S. Ryf, M. Spiegel, M. Gerber, and P. Boesiger. Myocardial tagging with 3D-CSPAMM. Journal of Magnetic Resonance Imaging, 16(3):320-325, 2002.

[15] J. Sainte-Marie, D. Chapelle, and M. Sorine. Data assimilation for an electro-mechanical model of the myocardium. In K.J. Bathe, editor, Second M.I.T. Conference on Computational Fluid and Solid Mechanics, pages 1801-1804, 2003.

[16] M. Sermesant, C. Forest, X. Pennec, H. Delingette, and N. Ayache. Deformable biomechanical models: Application to 4D cardiac image analysis. Medical Image Analysis, 7(4):475-488, 2003.

[17] N. Stergiopulos, B.E. Westerhof, and N. Westerhof. Total arterial inertance as the fourth element of the windkessel model. Am. J. Physiol., 276:H81-H88, 1999.

[18] D.R. Veronda and R.A. Westmann. Mechanical characterization of skin - finite deformation. Journal of Biomechanics, 3:114124,1970 . 